\title{
La centrale de la Jandula
}

1 a Société Canalizacion y Fuerzas del Guadalquivir (C. F. G.), une filiale de la Compania Anonima Mengemor, constitue avec cetle dernière el la Compania Sevillana de Electricidad, les entreprises d'électricité les plus importantes de l'Andalousie (Espagne méridionale).

Suivant un projel de son président, M. Mendoza, cetle Société a entrepris l'aménagement des forces motrices de Gua-
D'après le programme d'exploitation, la centrale de la Jandula ne travaille pas en hiver, de novembre à fin février; elle nu travaille qu'à faible charge de mars à fin juin et à pleine charge seulement dans la période de sécheressc, de juillet à fin octobre. C'esl d'après ce programme qu'à été projetée la centrale construite directement au pied du barrage auquel elle est adossée, disposition unique en Espagne jusqu'à ce jour. La salle des

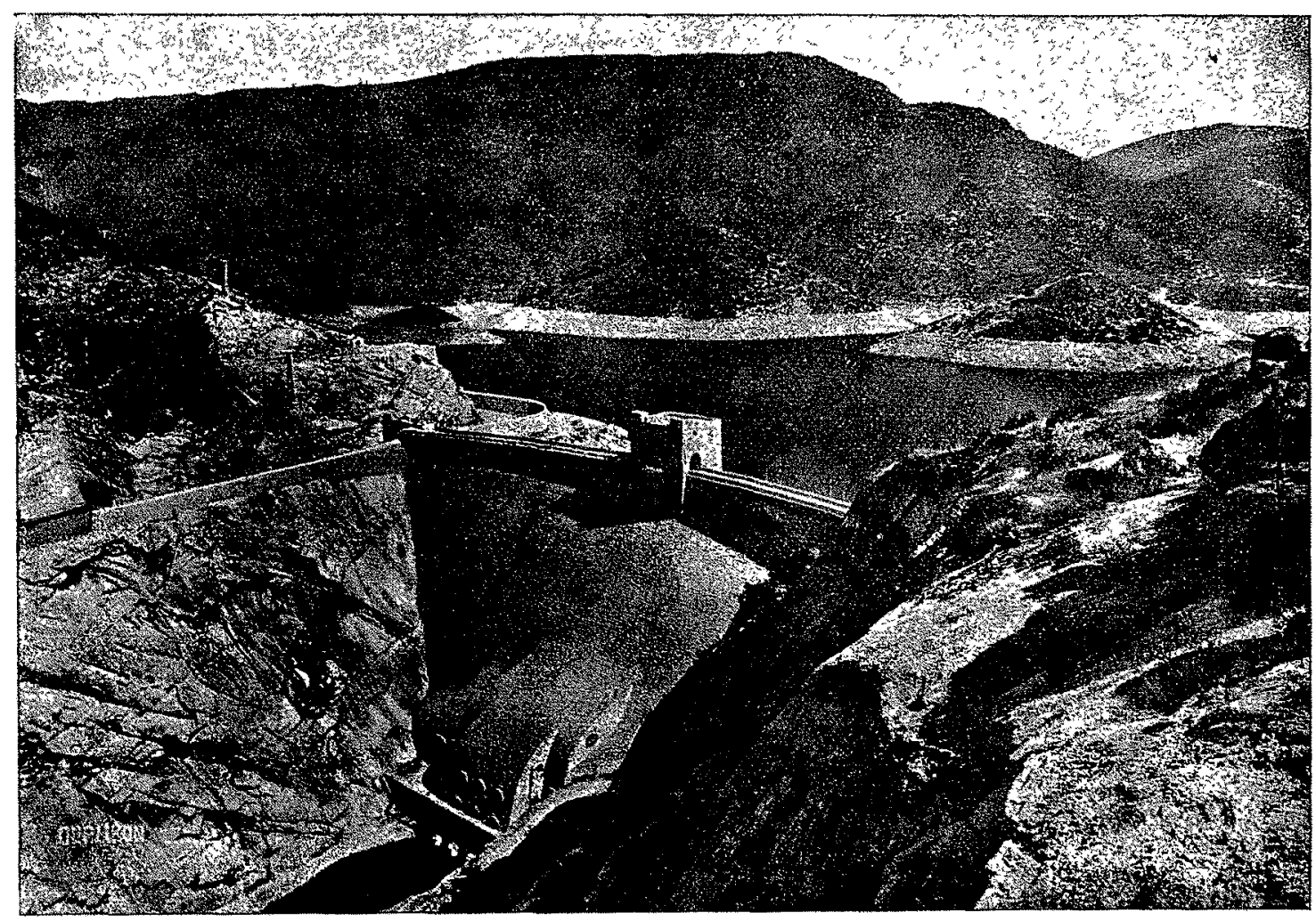

Fig. 1. - Vue du barrage avec la centrale adossée

dalquivir en dix chutes réparlies entre Séville el Cordoba. En liaison avec ces usines dont une est achevée jusqu'à ce jour, la C. F. G. a construit une centrale sur la Jandula, un des principaux amuents du haut Guadalviquir, dans la Sierra Morena. Un bassin d'accumulation de $350000000 \mathrm{~m}^{3}$ est formé par un barrage de gravité de 93 mètres de haut, d'une longueur de 22i) mètres à la couronne, dont la construction a nécessité environ $320000 \mathrm{~m}^{3}$ de béton.

Ce barrage, un des plus importants de l'Espagne, sert à régler lo débit du Guadalquivir pour canaliser ce fleuve et le rendre navigable jusqu'à Cordoba. II engendre en même temps, sur la Jandula, une chute que l'on utilise pour la production d'énergie électrique et l'irrigation. En Espagne, la plupart des cours d'eau ont un grand débit en hiver et occasionnent de nombreuses inondations, tandis qu'en été, par suite du manque de pluie, ils ont souvent un débit très faible et sont mème parfois à sec, de sorte qu'ils ne conviennent pas pour la navigation. Pour ces raisons la centrale de la Jandula a été prévue avec un bassin d'accumulation et de réglage qui doit pouvoir accumuler l'excédent d'eau en hiver et le laisser, en été, s'écouler peu à peu dans le Guadalquivir, de manière à rendre son débit plus uniforme, ce qui est d'unc importance capilale pour sa canalisation el

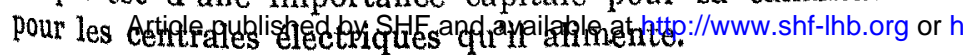

machines et les locaux pour l'appareillage à haute tension sont constitués par des voûtes avec des murs dont l'épaisseur atteint jusqu'à trois mètres, ce qui, vu le climat très chaud de l'Andalousie, ofire l'avantage que, même en plein été, la température intérieure de la centrale conserve une fraicheur agréable.

Il a été prévu un groupe de $3750 \mathrm{kVA}$ pour la période de charge réduite et deux groupes de $7500 \mathrm{kVA}$ pour le service d'été. Les turbines Francis à axe vertical ont été fournies par la Maison Th. Bell \& $\mathrm{C}^{\mathrm{le}}$, de Kriens. Elles répondent aux caractéristiques suivantes :

Groupes I et II :

Puissance............. $8620 \mathrm{ch}$

Débit maximum.......... $11000 \mathrm{l} / \mathrm{sec}$

Chute nette.......... $80-29 \mathrm{~m}$

Vitesse . . . . . . . . . . 375 tours/min

Vitesse d'emballement....... 773 tours/min

Groupe III :

Puissance............ $4100 \mathrm{ch}$

Débit maximum........ $50061 / \mathrm{sec}$

Chute nette........... $80-53 \mathrm{~m}$

Vitesse. .......... 500 tours $/ \mathrm{min}$

970 tours $/ \mathrm{min}$ 
Pour exécuter les groupes I et II pour une chute variable entre 80 et 29 mètres, sans crainte de mauvais rendement, ni de phénomènes de cavitation, il était nécessaire de prévoir chaque lurbine avec deux roues motrices facilement inlerchan- accouplement direct avec les turbines. Comme la distance entre la lurbine et l'alternateur est relalivement laible ll n'élaul pas nécessaire de recourr à un palier intermédiarre Los alternaleurs sont construils pour les données suivantes

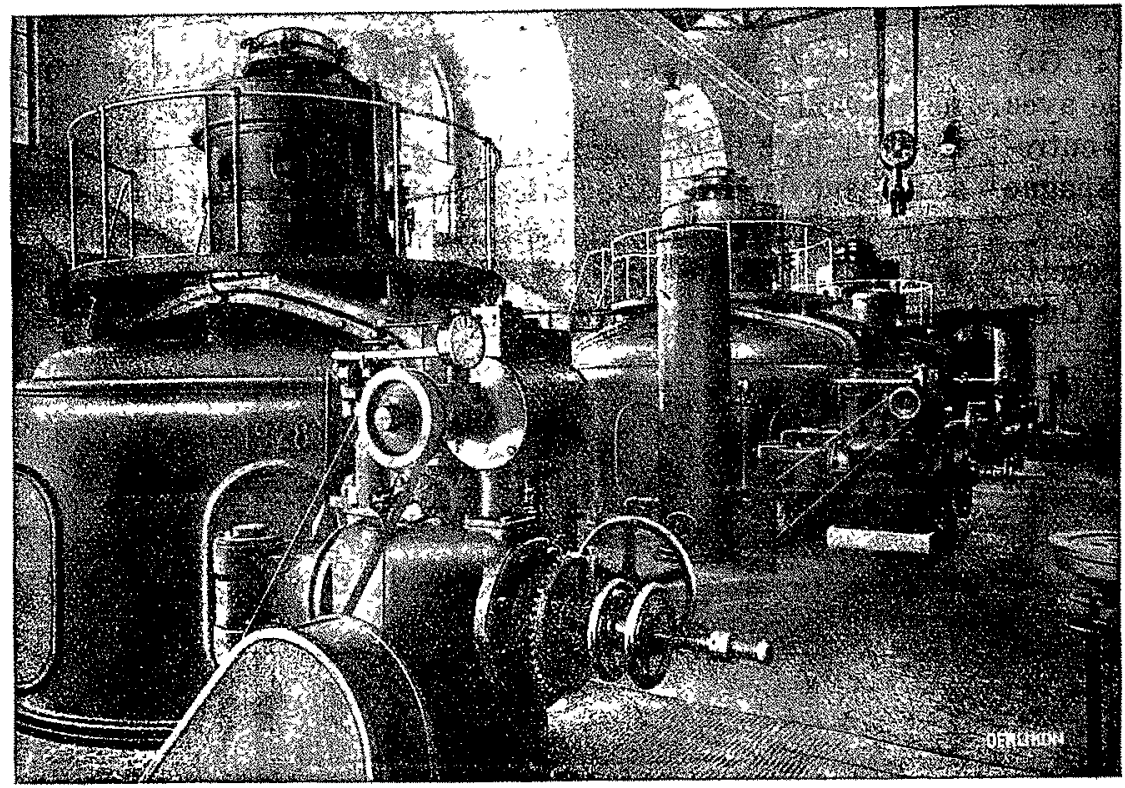

Fig. 2. - Salle des machines avec lalternateur de $3750 \mathrm{kVA}$ an premier plan et les alternateurs de $7500 \mathrm{kVA}$ au second plan

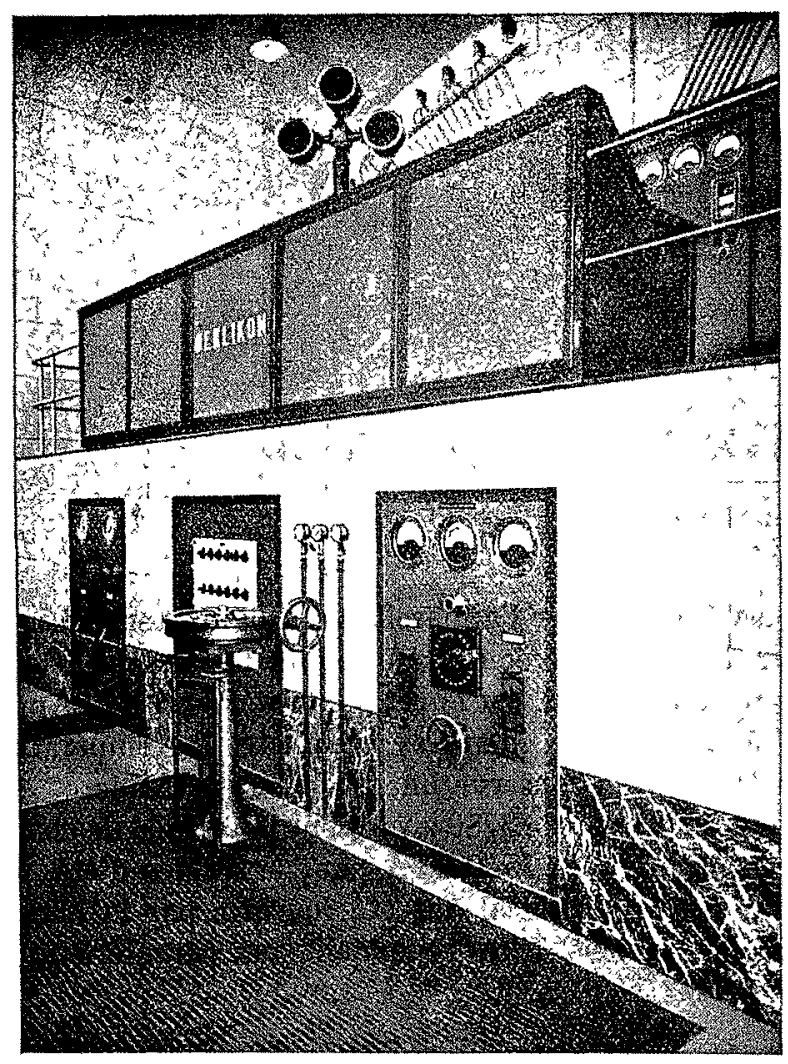

Fig. 4. - Pupitre de couplage pour les allernateurs et, au-dessous, tableau de couplage pour les services auxiliaires

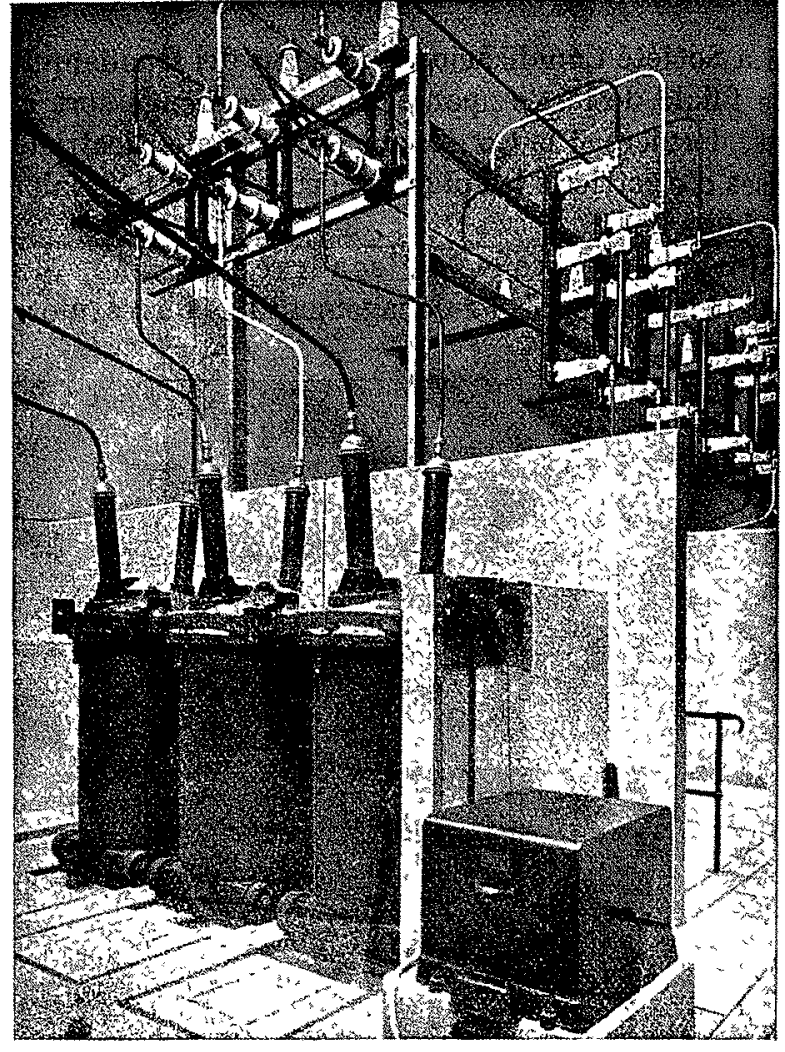

Fig 5. - Interrupteur à huilei dc grande puissance pour $66 \mathrm{kV}$, avec commande à distance par moteur; sectionneurs de ligne tripolaires et appareils de protection automatiques pour transformateurs de tension.

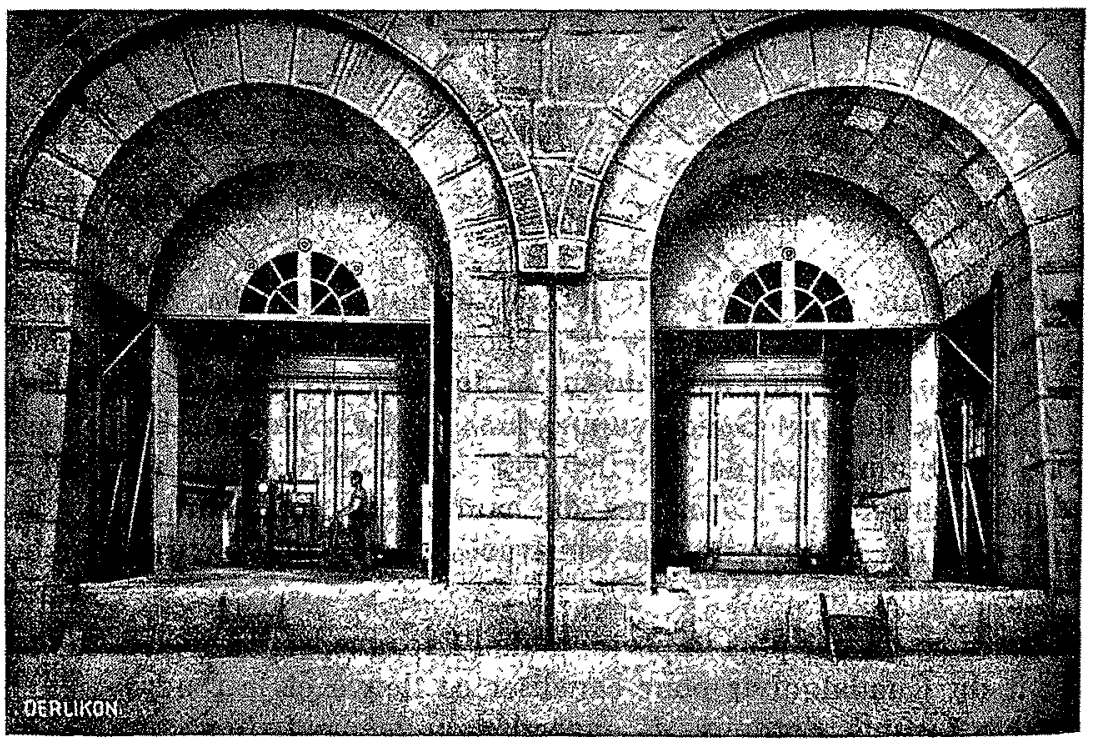

Fig. 3 - Vue à l'intérieur des cellules des transtormateurs de $11250 \mathrm{kVA}$. geables : l'une pour une chute de 80 à $48 \mathrm{~m}$., l'autre pour une chute de 48 à $29 \mathrm{~m}$. Les organes de fermeture des turbines sont des vannes sphériques à commande hydraulique. Cette commande est prévue de manière à pouvoir assurer encore la fermeture de la vanne, même sous une hauteur d'eau de $29 \mathrm{~m}$ seulement et avec l'appareil distributeur de la lurbine complètement ouvert.

Les trois alternaleurs sont de construction verlicale pour
Alternaleur I, 11 el 111

Nature du courant

Tension composée.

Fréquence.

$\cdots \ldots \ldots$

courant triphase 10000 volts 50 périodes/sec

\section{Alternateur I el II}

Allernateur III

Puissance ........... $7500 \mathrm{kVA} \quad 3750 \mathrm{kVA}$

Vitesse d'emballement.. $\quad \cdots \quad 375$ tours $/ \mathrm{min}$

$\mathrm{PD}^{2}$. $\quad . . . . . .99000 \mathrm{kmg}^{2}$

500 tous $/ \mathrm{min}$

970 tours/min

$25000 \mathrm{kmg}^{2}$ 
Le palier de butée qui repose sur lo croisillon supéricur, supporte the pression lolate (poids du rolor t poussée de l'eau) d'environ $66000 \mathrm{~kg}$ dans les groupes I et II et $36.400 \mathrm{~kg}$ au maximum dans le groupe III.

Chaque alternateur est équipé avec un frein pneumatique à l'aide duquel on peut l'arrêter en quelques minutes.

I.es trois machines sont du tvpe semi-fermé avec aspiration

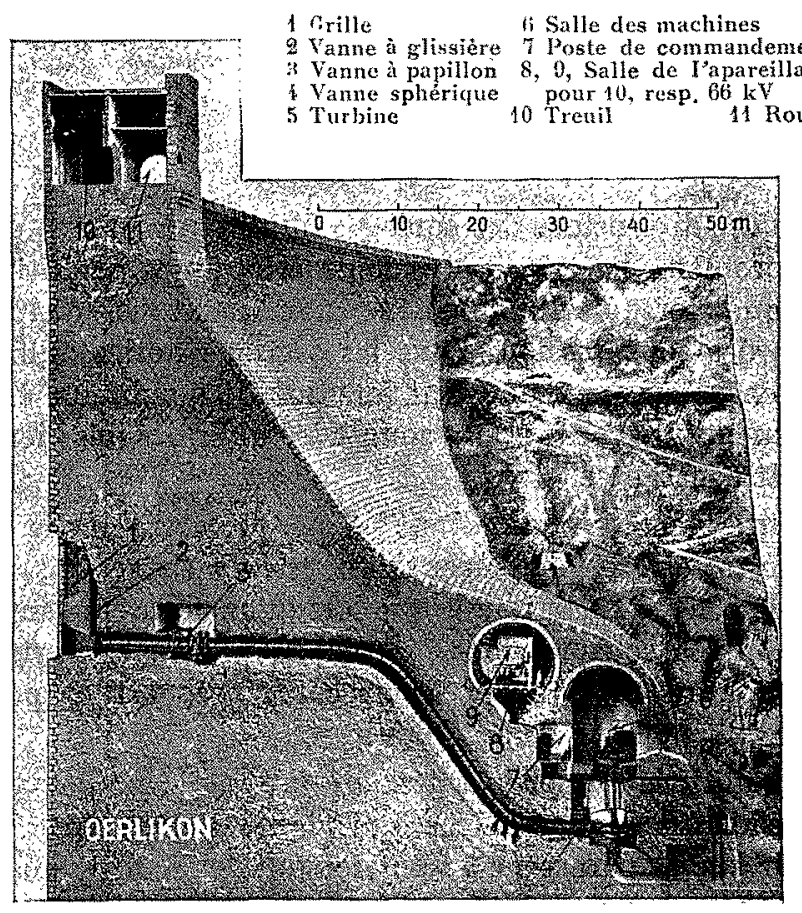

Fig. 6. Vue d'un modèle avec coupe du barrage et de la centrale, à échelle approximative.

de l'air de refroidissement de la chambre intermédiaire des turbines et refoulement de l'air chaud par une tubulure latérale dans la carcasse qui conduit directement à l'extérieur, à travers le mur du bâtiment. Les groupes sont donc complètement fermés du côté de la salle dus machines où l'air chaud ne peut pas pénétrer; ce qui est très important en raison du climat chaud de l'Espagne méridionale. Dans les canaux pour l'air chaud et l'air froid il a en outre été monté des clapets de protection, système Siebenmann, qui, en cas d'incendie, permettent de couper instantanément la circulation de l'air depuis la salle des machines, ou le poste de commandement et, par là, d'empêcher lo feu de se propager. Cette installation est facile à compléter par des extincteurs à acide carbonirue de Siebenmann. Les excitatrices sont montées directement sur les alternateurs qui sont en outre munis d'un dispositif de désexcitation rapide, systeme Oerlikon, permettant, en cas d'avarie, de supprimer immédiatement l'excitation des marhines.

Les trois alternateurs travaillent à travers des interrupteurs à huile convenablement dimensionnés, sur un système commun de barres omnibus auquel sont raccordés deux transformateurs élévateurs

Ces deux transfomateurs sont à refroidissement par circulalion d'huile. L'eau de refroidissement est prise dans un réservoir spécial, adossé au barrage, à une hauteur d'environ 30 mètres. Les transformateurs sont construits pour les caractéristiques suivantes :

Tension primaire $\ldots \ldots \ldots \ldots \ldots \ldots \ldots$

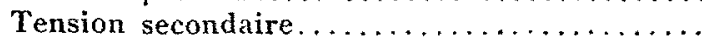

Fréquence.........................

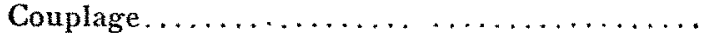

$11250 \mathrm{kVA}$

10000 volts

70000 volts

$50 \mathrm{pér}$. $/ \mathrm{sec}$

En passant au couplage étoile, on pourra au besoin porter la tension secondaire à 121000 volts.

Les réfrigérants d'huile des transformateurs sont équipés avec. des avertisseurs éprouvés, système Oerlikon, qui signalent les interruptions dans la circulation de l'eau et de l'huile. Is sont en outre protégés par des appareils Buchholz et munis de thermomètres à distance.

Le courant triphasé transformé à 70000 volts est amené à travers des interrupteurs à huile de grande puissance, capables de couper des courts-circuits de $350000 \mathrm{kVA}$, aux barres omnibus à haute tension, d'oì partent deux lignes qui alimentent le réseau de transmission de la Canalizacion y Fuerzas del Guadalquivir.

Tous les interrupteurs à huile sont pourvus d'une commande à distance par moteur, qui permet de les actionner depuis lo poste de commandement; il en est de même du dispositif de désexcitation rapide des alternateurs. Le poste de commandement est logé dans une niche d'où il domine la salle des machines.

Pour l'alimentation des services auxiliaires, il est prévu deux transformateurs de $65 \mathrm{kVA}, 10000 / 200 / 127$ volts, ainsi qu'une batterie d'accumulateurs de 180 ampères-heure avec des groupes de charge, réducteur, etc.

Un pont roulant électrique de 30 tonnes dans la salle des machines et une installation pour le filtrage et la cuisson'de l'huile complètent les fournitures des Ateliers de Construction Oerlikon.

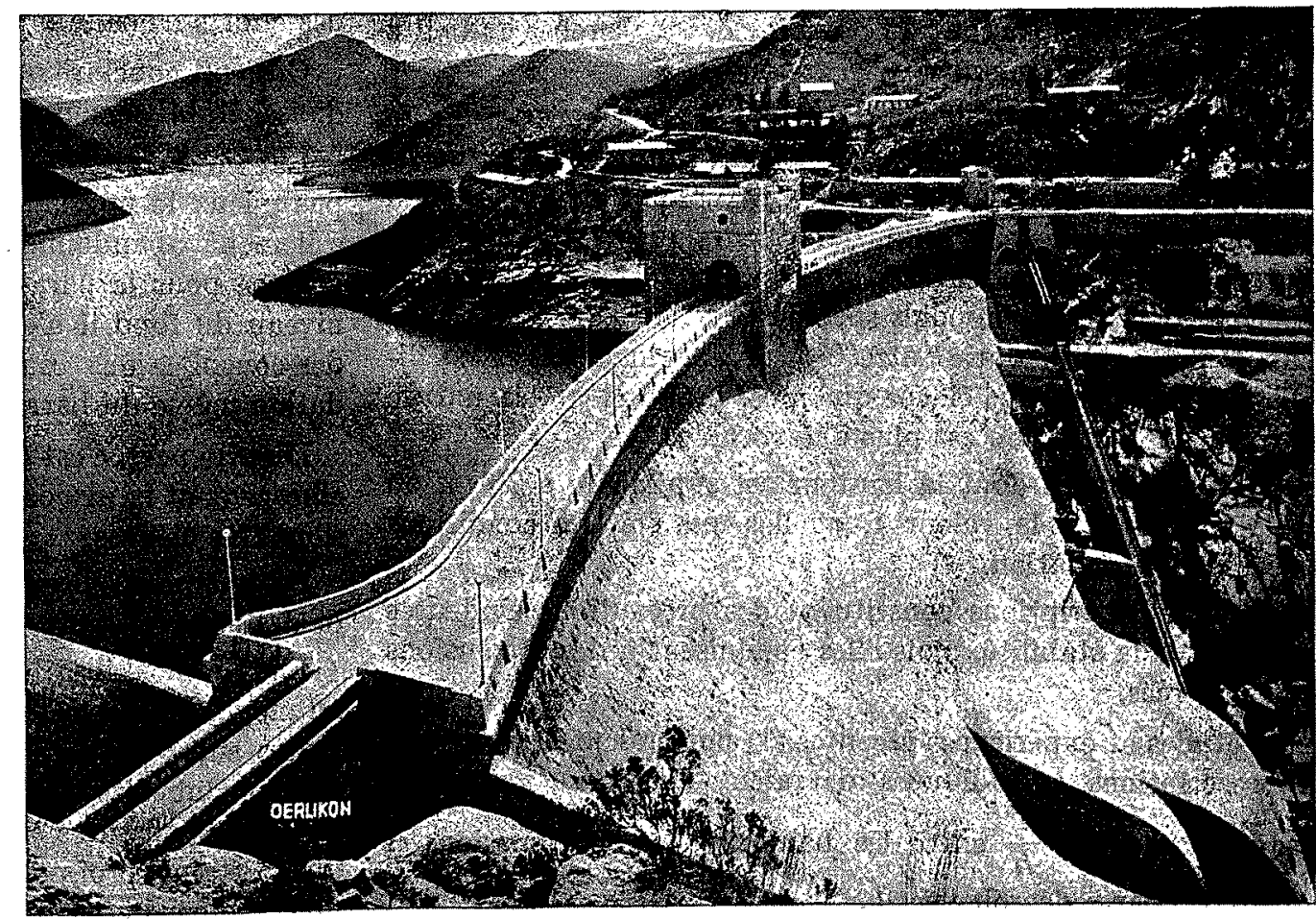

Fig. 7.-- Vue du barrage et de la plus grande partic du bassin d'accumulation.
Cette centrale qui se trouve régulièrement en service depuis l'été 1929, travaille à l'entière satisfaction du client. 